\title{
Abstract Author Index
}

A

Aibiki, M., PO-008

Akamatsu, M., PO-015

Akimoto, H., PO-012

Al-Dorzi, H., PO-026

Al-Jabbary, A., PO-026

Al-Subaie, N., PO-040

Ameloot, K., PO-025

Andrews, P., PO-007, PO-010, PO-038

Asai, Y., OC-002

Atsumi, T., OC-002

\section{B}

Bartos, J., PO-037

Baumgard, M., PO-041

Belohlavek, J., PO-001

Berger, F., PO-028, PO-029, PO-033

Bestle, M., OC-005

Beylin, M., PO-030

Biasucci, D.G., PO-002

Bill, P., PO-031

Billot, L., OC-006

Boned, S., PO-036

Boos, L., PO-016

Borgquist, O., PO-040

Bullock, R., OC-012

\section{C}

Cai, H., OC-011

Cattaneo, G.F.M., PO-016

Chakkarapani, E., PO-020

Chen, C., OC-011

Christensen, H., OC-005

Clark, C., PO-007

Clodi, C., PO-039

Colbourne, F., OC-009

Coleman, R., PO-041

Collignon, O., PO-035

Conterato, M., PO-027

Conti, G., PO-002

Cozzi-Lepri, A., OC-005

Cranshaw, J., PO-040

Cronberg, T., OC-001, OC-004, PO-017, PO-040

Crossley, S., PO-007

Csernyik, E., PO-041

\section{D}

Dankiewicz, J., PO-021, PO-034

De Deyne, C., OC-003, PO-025

De Luca, D., PO-002

Debaty, G., PO-037

Deeb, A.M., PO-026

Dens, J., OC-003, PO-025

Devaux, Y., PO-035

Dhaliwal, R., PO-027

Dickey-White, H., PO-041

Dietrich, D., OC-012

Dingley, J., PO-020

Dörfler, A., OC-007

Dragancea, I., OC-004
Dragancea, I.V., PO-017

Drummond, E., PO-041

Duez, C., PO-019

Dupont, M., PO-025

$\mathbf{E}$

Ebermann, N., PO-029

Ebner, F., PO-024

Ellenberger, D., PO-041

Erlinge, D., PO-009

Ernstrom, K., PO-003

Ettl, F., PO-039

F

Feldmann, M., PO-033

Feldbæk Nielsen, J., PO-019

Flores, A., PO-036

Flynn, L., PO-010, PO-038

Foerster, K., PO-016

Friberg, H., OC-001, OC-004, PO-017, PO-034, PO-040

Fukuda, M., PO-012

\section{G}

Gaieski, D.F., PO-030

Garlisi, A., PO-041

Garrett, F., PO-018

Genbrugge, C., OC-003, PO-025

Genovese, O., PO-002

Gill, H., PO-020

Gjedsted, J., PO-004

Glover, G., PO-040

Gotberg, M., PO-009

Goyal, M., PO-030

Grassmann, D., PO-039

Grejs, A., PO-004

Grossestreuer, A., PO-030

Grotta, J., PO-003

H

Hammond, N.E., OC-006

Hase, M., OC-002

Hayashi, N., OC-008

Hayton, J., PO-007

Hemmen, T., PO-003, PO-006

Hifumi, T., OC-008

Hirakawa, E., PO-022, PO-023

Horn, J., OC-001, PO-017

Huptych, M., PO-001

Huttner, H.B., OC-007

I

Ibara, S., PO-022, PO-023

Inoue, K., PO-015

$\mathbf{J}$

Janata, A., PO-039

Jans, F., OC-003

Jaworski, A., PO-030
Jensen, J.-U., OC-005

Jia, X., OC-011

Johansen, M.E., OC-005

Johansson, P., OC-005

Johnsen, B., PO-019

Johnsson, J., PO-021

K

Kanno, M., PO-013

Karlsson, V., PO-034

Kashiwagi, M., PO-015

Kawase, K., PO-005

Keller, E., PO-032

Kern, K.B., PO-034

Keuler, A., PO-016

Kikuchi, S., PO-008

Kim, D.H., OC-012

Kirkegaard, H., PO-004, PO-014, PO-019

Kjær, J., OC-005

Kjaergaard, J., OC-001

Klein, J., PO-041

Kloska, S., OC-007

Kobata, H., PO-012

Kollmar, R., OC-007

Krauß, A., PO-028

Kuiper, M., PO-017

Kulstad, E., PO-018

Kuroda, Y., OC-008, OC-012

L

Lassen, J.F., PO-004

Lilja, G., OC-001

Linhart, A., PO-001

Liu, X., PO-020

Luk, J., PO-041

Lundgren, J., OC-005

Lybeck, A., OC-004

Lyden, P., PO-003, PO-006

M

Ma, Y., OC-009

MacDougall, M., PO-007

Maekawa, T., OC-008

Magnet, I.A.M., PO-039

Matsui, T., PO-011

Matsumoto, H., PO-008

Matsuura, T., PO-037

Maybhate, A., OC-011

McDevitt, W., PO-031

McKnite, S., PO-037

McLatchie, R., PO-007

Meckel, S., PO-016

Meex, I., OC-003, PO-025

Meler, P., PO-036

Mikkelsen, M., PO-030

Miyazaki, S., PO-011

Mizoue, R., OC-010, PO-005

Mochner, J., PO-028

Mohr, T., OC-005

Molina, C.A., PO-036

Møller, K., OC-005 
Morimatsu, H., OC-010, PO-005

Morimura, N., OC-002

Morris, K., PO-031

Muchada, M., PO-036

Mullens, W., PO-025

Müller, A., PO-032

Murata, S., PO-015

Myburgh, A., OC-006

Myburgh, J., OC-006

Mysore, J., OC-006

Nagao, K., OC-002

Nagao, S., OC-008

Nara, S., OC-002

Nielsen, N., OC-001, OC-004, PO-017, PO-021, PO-034, PO-040

Niesen, W.-D., PO-016

Nilsson, F., OC-001

Notghi, L., PO-031

Okasora, K., PO-015

Ooba, C., PO-015

Ostrowski, S., OC-005

$\mathbf{P}$

Pagola, J., PO-036

Patterson, D., PO-018

Pedersen, H.P., OC-005

Pellis, T., OC-001, OC-004

Perman, S., PO-030

Piastra, M., PO-002

Picconi, E., PO-002

Pizza, A., PO-002

Poulsen, L., OC-005

\section{$\mathbf{R}$}

Raman, R., PO-003

Rapp, K., PO-003

Rees, J., PO-037

Reid, J., PO-007

Rhodes, J., PO-010, PO-038

Ribo, M., PO-036
Richi, H., PO-026

Rodriguez-Luna, D., PO-036

Rubiera, M., PO-036

S

Sabir, H., PO-020

Sakamoto, T., OC-002

Sanjuan, E., PO-036

Sato, S., OC-010, PO-005

Saxena, M., OC-006

Schlick, K., PO-006

Schmitt, K., PO-028, PO-029, PO-033

Schober, A., PO-039

Scholefield, B., PO-031

Schriefl, C., PO-039

Schumacher, M., PO-016

Schwab, S., OC-007

Seder, D., PO-034

Sero, L., PO-036

Sertznig, C., PO-035

Shah, M., PO-016

Shanley, P., PO-018

Shigehara, S., PO-015

Shimizu, M., PO-013

Shirasu, A., PO-015

Silvestro, D., PO-041

Skalicka, H., PO-001

Sloth, E., PO-004

Smid, O., PO-001

Smit, E., PO-020

Stammet, P., PO-035

Staykov, D., OC-007

Sterz, F., PO-039

Suehiro, E., OC-012

Sugie, A., PO-012

T

Tahara, Y., OC-002

Takata, K., OC-010

Takeba, J., PO-008

Takeda, Y., OC-010, PO-005

Tamai, H., PO-015

Tanabe, T., PO-015

Taylor, C., OC-006

Thompson, K., OC-006
Thoresen, M., PO-020

Thormar, K., OC-005

Tokuhisa, T., PO-022, PO-023

Tong, G., PO-028, PO-029, PO-033

Tønnesen, E., OC-005

Tooley, J., PO-020

$\mathbf{U}$

Ullén, S., PO-017

Umakoshi, K., PO-008

Unger, B.T., PO-034

V

Van Tornhout, E., OC-003

Vanhove, J., OC-003

Vedia, B., PO-018

Volbers, B., OC-007

\section{W}

Wagner, M., PO-039

Walden, A., PO-040

Walker, C., PO-033

Warenits, A.-M., PO-039

Waterston, T., PO-031

Weber, S., PO-029

Weihs, W., PO-039

Werer, C., PO-035

Wetterslev, J., OC-001, PO-017

Windsor, D., PO-020

Wise, M., PO-040

Wollersheim, S., PO-028

Woods, C., PO-041

Wowk, S., OC-009

$\mathbf{Y}$

Yamashita, S., OC-008

Yamazaki, S., PO-015

Yannopoulos, D., PO-037

Yokobori, S., OC-012

Yokota, H., 002, 012

Yoritsune, E., PO-012

Young, P., OC-006

$\mathbf{Z}$

Zughaft, D.J., PO-009 